\title{
Prospective Multicenter Study of the Challenges Inherent in Using Large Cell-Type Stents for Bilateral Stent-in-Stent Placement in Patients with Inoperable Malignant Hilar Biliary Obstruction
}

\author{
Min Jae Yang ${ }^{1}$, Jin Hong Kim ${ }^{1}$, Jae Chul Hwang ${ }^{1}$, Byung Moo Yoo ${ }^{1}$, Sang Hyub Lee ${ }^{2}$, Ji Kon Ryu ${ }^{2}$, Yong-Tae Kim² ${ }^{2}$ Sang \\ Myung Woo ${ }^{3}$, Woo Jin Lee ${ }^{3}$, Seok Jeong ${ }^{4}$, and Don Haeng Lee ${ }^{4}$ \\ ${ }^{1}$ Department of Gastroenterology, Ajou University School of Medicine, Suwon, ${ }^{2}$ Department of Internal Medicine and Liver Research Institute, \\ Seoul National University College of Medicine, Seoul, ${ }^{3}$ Center for Liver Cancer, National Cancer Center, Goyang, and ${ }^{4}$ Digestive Disease Center, \\ Department of Internal Medicine, Inha University School of Medicine, Incheon, Korea
}

Background/Aims: Although endoscopic bilateral stent-instent placement is challenging, many recent studies have reported promising outcomes regarding technical success and endoscopic re-intervention. This study aimed to evaluate the technical accessibility of stent-in-stent placement using large cell-type stents in patients with inoperable malignant hilar biliary obstruction. Methods: Forty-three patients with inoperable malignant hilar biliary obstruction from four academic centers were prospectively enrolled from March 2013 to June 2015. Results: Bilateral stent-in-stent placement using two large cell-type stents was successfully performed in $88.4 \%$ of the patients (38/43). In four of the five cases with technical failure, the delivery sheath of the second stent became caught in the hook-cross-type vertex of the large cell of the first stent, and subsequent attempts to pass a guidewire and stent assembly through the mesh failed. Functional success was achieved in all cases of technical success. Stent occlusion occurred in $63.2 \%$ of the patients (24/38), with a median patient survival of 300 days. The median stent patency was 198 days. The stent patency rate was $82.9 \%$, $63.1 \%$, and $32.1 \%$ at 3,6 , and 12 months postoperatively, respectively. Endoscopic re-intervention was performed in 14 patients, whereas 10 underwent percutaneous drainage. Conclusions: Large cell-type stents for endoscopic bilateral stent-in-stent placement had acceptable functional success and stent patency when technically successful. However, the technical difficulty associated with the entanglement of the second stent delivery sheath in the hook-cross-type vertex of the first stent may preclude large cell-type stents from being considered as a dedicated standard tool for stent-in-stent placement. (Gut Liver 2018;12:722-727)

Key Words: Cholangiopancreatography, endoscopic retrograde; Klatskin tumor; Cholestasis, intrahepatic; Self expandable metallic stents

\section{INTRODUCTION}

Endoscopic biliary drainage using self-expandable metal stents (SEMSs) is the principal palliative procedure for treating inoperable malignant hilar biliary obstruction. ${ }^{1-3}$ With the recent advancements in SEMS technology, bilateral biliary drainage has become increasingly popular. ${ }^{4-16}$ This procedure aims to maintain an effective drainage of $>50 \%$ of the liver volume and ultimately offer long-term palliation. ${ }^{17-19}$ Better rates of survival $^{20}$ and stent patency ${ }^{21-23}$ and fewer re-interventions ${ }^{23}$ have been reported with bilateral stenting than with unilateral stenting.

Stent-in-stent (SIS) placement is a well-established endoscopic technique for bilateral biliary drainage. The major limitation of the SIS technique, however, is its technical difficulty, especially when passing the guidewire through the wire mesh of the indwelling first stent and delivering the second stent system to the desired contralateral intrahepatic duct. ${ }^{3,24}$ Furthermore, subsequent endoscopic re-interventions are as technically challenging as the initial procedure. ${ }^{15}$ Despite these widely known limitations, most feasibility studies on the efficacy of the SIS technique using dedicated SEMSs, such as an open-weave, cross-wired metal stent and a uniform large cell-type stent, have reported near-perfect technical success. ${ }^{5,8-10,15}$ Therefore, in this

Correspondence to: Jin Hong Kim

Department of Gastroenterology, Ajou University School of Medicine, 164 World cup-ro, Yeongtong-gu, Suwon 16499, Korea

Tel: +82-31-219-6937, Fax: +82-31-219-5999, E-mail: jinhkim@ajou.ac.kr

Received on October 23, 2017. Revised on January 11, 2018. Accepted on March 9, 2018. Published online August 9 , 2018

pISSN 1976-2283 eISSN 2005-1212 https://doi.org/10.5009/gnl17468

@) This is an Open Access article distributed under the terms of the Creative Commons Attribution Non-Commercial License (http://creativecommons.org/licenses/by-nc/4.0) which permits unrestricted non-commercial use, distribution, and reproduction in any medium, provided the original work is properly cited. 
multicenter study, we aimed to evaluate the technical feasibility of SIS placement using large cell-type stents in patients with inoperable malignant hilar biliary obstruction.

\section{MATERIALS AND METHODS}

\section{Patients}

From March 2013 to June 2015, patients with malignant hilar biliary obstruction who met the inclusion criteria were prospectively enrolled at four academic centers in Korea. The inclusion criteria were as follows: (1) >20 years of age; (2) Bismuth type II or higher malignant hilar biliary obstruction caused by cholangiocarcinoma or gallbladder cancer; and (3) unable to undergo curative resection because of locally advanced cancer, metastasis, or high surgical risk. The exclusion criteria included a history of prior stent placement or biliary surgery other than cholecystectomy or liver atrophy and uncontrolled coagulopathy (international normalized ratio $>1.5$ or platelet count $<50,000 / \mathrm{mm}^{3}$ ). Written informed consent was obtained from all patients. The study was approved by each institution's ethics review board (AJIRB-DEV-DE3-12-351, 1312-013-540, NCCCTSMD-011, MD20130003) and was registered at cris.nih.go.kr (KCT0002198).

\section{Endoscopic procedure}

The Niti-S ${ }^{\mathrm{TM}}$ large cell-type biliary stent (Taewoong Corp., Seoul, Korea) is a nitinol-based metal stent that contains large uniform mesh cells (6 mm square) for easy passage of the second stent (Fig. 1A). It consists of eight nitinol wires for optimal radial force $(4.9 \mathrm{~N})$, which are thicker $(0.203 \mathrm{~mm})$ than conventional biliary stents, and a hook and cross-mesh structure for low axial force $(0.0 \mathrm{~N})$. The stent has three radiopaque markings at each end and two in the midsection. An 8-F stent delivery system is used.
Computed tomography (CT) and/or magnetic resonance cholangiopancreatography-targeted selective drainage was performed before endoscopic retrograde cholangiopancreatography (ERCP). Following the placement of two guidewires (0.035-inch Jagwire $^{\mathrm{TM}}$ [Boston Scientific, Marlborough, MA, USA], 0.035inch Tracer Metro ${ }^{\circledR}$ [Cook Medical, Bloomington, IN, USA], or 0.025-inch VisiGlide [Olympus Corp., Tokyo, Japan]) into the targeted bilateral intrahepatic branches across the hilar stricture, carbon dioxide cholangiography was performed first by injecting 10 to $20 \mathrm{~mL}$ of carbon dioxide to prevent post-procedure cholangitis in the contaminated and undrained ducts. If needed, contrast was injected to enhance the bile duct. The first stent was then inserted into either the left or right hepatic duct, depending on which duct was more difficult to access. Balloon dilation of the first stent was performed if the first stent was inadequately expanded or kinked. Thereafter, the guidewire was withdrawn to the hilar level and reinserted into the contralateral side via the mesh of the first stent, referencing the other landmark guidewire. If the guidewire could not be advanced into the contralateral intrahepatic duct through the first stent mesh, a J-shaped hydrophilic guidewire (Radifocus ${ }^{\circledR}$; Terumo, Tokyo, Japan) and a catheter with an adjustable tip angle (TRUEtome ${ }^{\mathrm{TM}}$; Boston Scientific or Swing Tip ${ }^{\circledR}$; Olympus Corp.) was used. The delivery system of the second stent was then advanced over the guidewire; however, if the delivery system was unable to be passed, dilation of the first stent was performed, and another attempt was made to pass the guidewire through the mesh cell. Subsequently, the contralateral stricture and first stent mesh were dilated using either a 6- to 8-mm balloon catheter (Hurricane ${ }^{\mathrm{TM}}$ biliary balloon catheter; Boston Scientific), a 6- or 7-F bougie (Soehendra ${ }^{\circledR}$ Biliary Dilation Catheter; Cook Medical), or a Soehendra ${ }^{\circledR}$ Stent Retriever (Cook Medical) over the guidewire. Finally, the second stent was placed in a Y-shaped position, considering the distal level of the stricture (Fig. 1B).

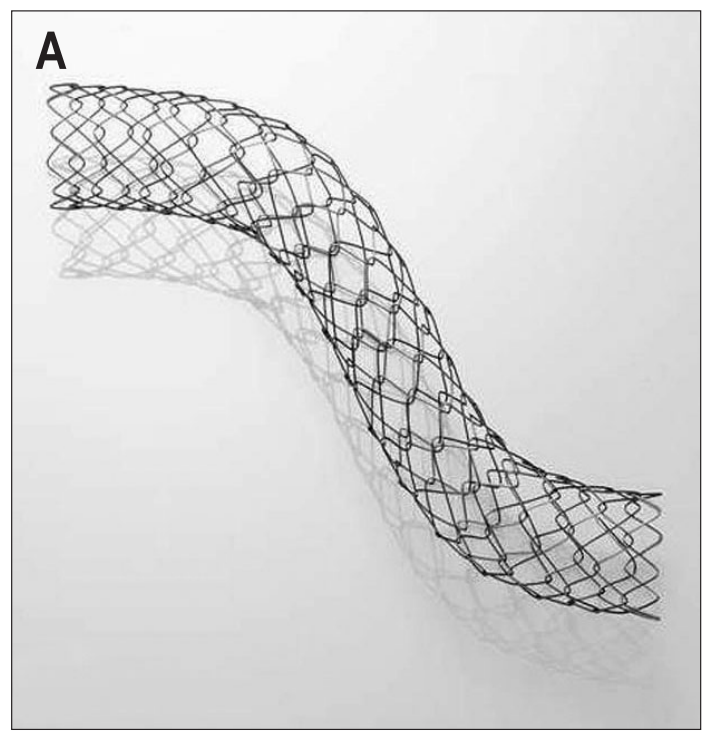

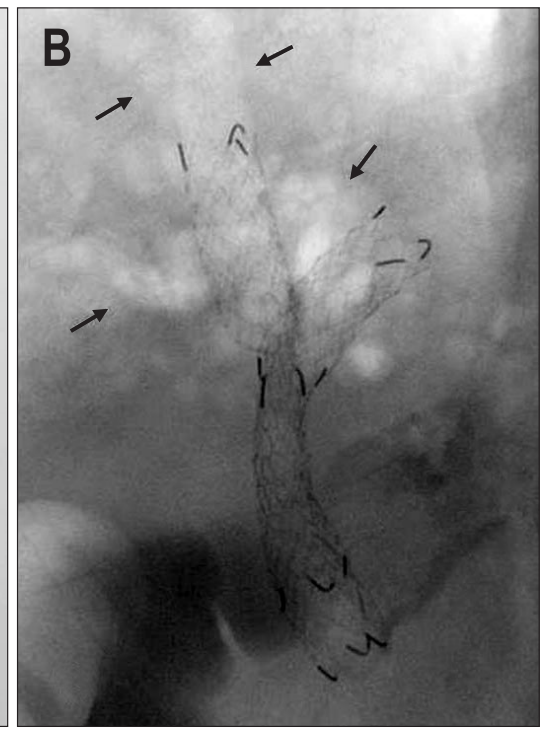

Fig. 1. (A) The Niti-S ${ }^{\mathrm{TM}}$ large celltype biliary stent (Taewoong Corp.). The hook-cross-type vertex of the mesh cell. (B) Carbon dioxide cholangiography (arrows) and bilateral stent-in-stent placement using large cell-type stents. 


\section{Measured outcomes and follow-up}

Technical success was defined as the passage of bilateral large cell-type stents across the stricture using the SIS technique, in a single attempt. Functional success was defined as a reduction in the bilirubin level back to normal or to $75 \%$ of the pre-treatment level within the first month after the procedure. ERCP-related adverse events and grade of severity were defined according to the 1991 consensus criteria ${ }^{25}$ and the Tokyo guideline. ${ }^{26,27}$ Early and late adverse events were defined as any stent-related adverse event, including stent occlusion, within or after 30 days of stent placement, respectively. Stent occlusion was suspected if recurrent jaundice and/or clinical signs of acute cholangitis were observed and were confirmed by intrahepatic bile duct dilation identified on ultrasonography, CT, percutaneous cholangiography, or ERCP. Tumor overgrowth was defined when the obstruction level was identified at the proximal or distal end of a stent on CT and/or ERCP and contrast enhancement was seen at the obstruction level on CT. Biliary sludge was defined when contrast enhancement was not noted at the obstruction level on CT and fluoroscopic images showed intraluminal clearance of the stent after a biliary balloon sweep. Tumor ingrowth was defined when the obstruction level was detected inside of the stent lumen and enhancing tissue was seen within the stent on CT or when fluoroscopic images did not show intraluminal clearance of the stent with a biliary balloon sweep. Outpatient follow-up was conducted via liver function tests at 1 -week post-procedure and then monthly for a period of 1-year post-procedure. Follow-up data collection was terminated at the date of death

Table 1. Baseline Characteristics of Patients $(n=43)$

\begin{tabular}{lc}
\multicolumn{1}{c}{ Characteristic } & Value \\
\hline Age, yr & $72.9 \pm 9.1$ \\
Sex, male/female & $22 / 21$ \\
Cholangiocarcinoma/GB cancer & $27(62.8) / 16(37.2)$ \\
Bismuth type & \\
II & $14(32.6)$ \\
IIIa & $17(39.5)$ \\
IIIb & $1(2.3)$ \\
IV & $11(25.6)$ \\
Karnofsky Performance Score & $76.6 \pm 9.1$ \\
Adjuvant treatment & \\
Chemotherapy & $12(27.9)$ \\
Radiotherapy & $2(4.7)$ \\
Both & $1(2.3)$ \\
Total bilirubin level, mg/dL & $8.9 \pm 6.4$ \\
Follow-up period, day & $184(38-732)$ \\
\hline
\end{tabular}

Data are presented as mean \pm SD, number (\%), or median (range). $\mathrm{GB}$, gallbladder. or at the 1-year follow-up visit of the last enrolled patient (May 2016). Patients were lost to follow-up when no contact was made with the patient within 3 months after the stent insertion.

\section{Statistical analyses}

For sample size calculation, we set the expected technical success rate of SIS placement using large cell-type stents to $96 \%{ }^{9}$ with an $80 \%$ threshold rate with type I error of 0.05 and a statistical power of 0.8. The estimated sample size was 40 patients, assuming a 10\% dropout rate. Mean and standard deviation were used to describe continuous variables, while percentages were used for categorical variables. The Kaplan-Meier method was used to assess the median time to recurrent biliary obstruction and overall patient survival. Statistical analyses were performed in an intention-to-treat manner, using SPSS version 18.0 (SPSS Inc., Chicago, IL, USA).

\section{RESULTS}

A total of 43 patients with inoperable malignant hilar biliary obstruction who met the inclusion criteria were enrolled in the study (Table 1). The causes of hilar biliary obstruction included cholangiocarcinoma in 27 patients and gallbladder carcinoma in 16 patients. The types of hilar obstruction, as based on the Bismuth-Corlette classification, were II in 14 patients, IIIa in 17 patients, IIIb in one patient, and IV in 11 patients. Fifteen patients (34.9\%) underwent palliative chemotherapy or radiotherapy for carcinoma.

Table 2. Overall Outcomes of Endoscopic Bilateral Stent-in-Stent Placement Using Large Cell-Type Stents for Inoperable Malignant Hilar Biliary Obstruction

\begin{tabular}{|c|c|}
\hline Outcomes & Data \\
\hline Technical success & $38 / 43(88.4)$ \\
\hline Technical failure & $5 / 43(11.6)$ \\
\hline Bismuth type II/IIIa/IIIb/IV & $0 / 2 / 1 / 2$ \\
\hline $\begin{array}{l}\text { Failure in guidewire reinsertion via first } \\
\text { stent mesh }\end{array}$ & 1 \\
\hline $\begin{array}{l}\text { Second stent delivery passage failure via } \\
\text { first stent mesh }\end{array}$ & 4 \\
\hline Functional success & $38 / 43(88.4)$ \\
\hline Stent occlusion & $24 / 38(63.2)$ \\
\hline Tumor ingrowth & 17 \\
\hline Tumor ingrowth and overgrowth & 4 \\
\hline Biliary sludge & 3 \\
\hline Overall stent patency & $198(68-328)$ \\
\hline Patency rate at 3/6/12 mo, \% & $82.9 / 63.1 / 32.1$ \\
\hline Overall patient survival, day & $300(144-455)$ \\
\hline Endoscopic/percutaneous re-intervention, n (\%) & $14(58.3) / 10(41.7)$ \\
\hline
\end{tabular}




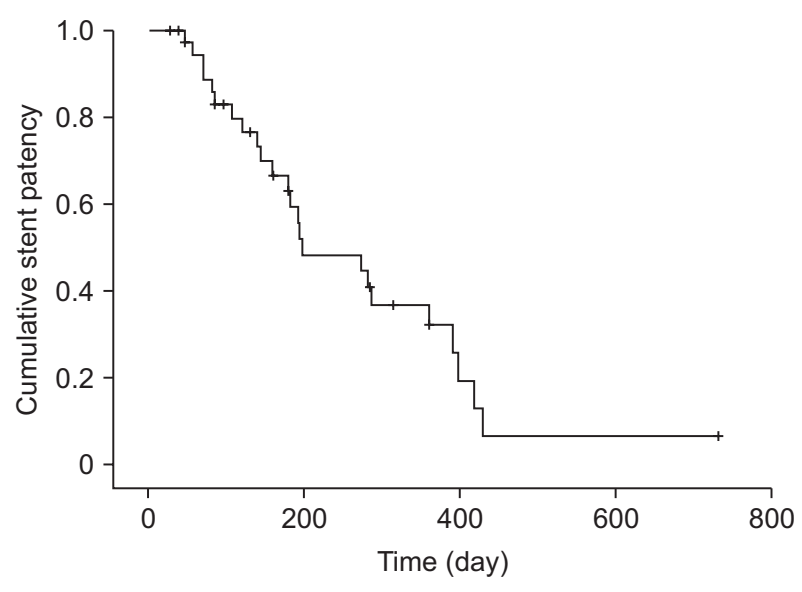

Fig. 2. Kaplan-Meier curve of cumulative stent patency.

Stent-related outcomes are summarized in Table 2. Bilateral SIS placement using two large cell-type stents was successfully performed in $88.4 \%$ of patients (38/43). There were five cases of technical failure in which the Bismuth types were IIIa ( $\mathrm{n}=2)$, IIIb $(n=1)$, or IV $(n=2)$. In the first case of failure, after placement of the first stent, the first guidewire could not be reinserted into the contralateral hepatic duct via the mesh of the first stent, despite the use of a hydrophilic guidewire and a catheter with an adjustable tip angle. In the remaining four cases of technical failure, the delivery sheath of the second stent could not be advanced through the first stent's mesh, despite the guidewire being successfully placed in the contralateral intrahepatic duct via the mesh of the first stent. In one of four cases, bilateral stenting was finally achieved using a different type of stent for the second stent with a 6-F delivery sheath. In the other three cases, the delivery sheath of the second stent was caught in the hook-cross-type vertex of the first stent's mesh cell; hence, even the 6-F bougie dilator and 7-F Soehendra ${ }^{\circledR}$ Stent Retriever could not be advanced outside the first stent's mesh.

Functional success was achieved in all 38 patients for whom technical success was achieved. Stent occlusion occurred in $63.2 \%$ of patients (24/38), with a median patient survival of 300 days (interquartile range, 144 to 455 days), and cases of occlusion were caused by tumor ingrowth $(\mathrm{n}=17)$, ingrowth combined with overgrowth $(n=4)$, or biliary sludge $(n=3)$. Estimated median stent patency based on Kaplan-Meier analysis was 198 days (interquartile range, 68 to 328 days) (Fig. 2). The stent patency rate was $82.9 \%$ at 3 months, $63.1 \%$ at 6 months, and $32.1 \%$ at 1 year. Endoscopic re-intervention was performed in 14 patients (58.3\%) with stent occlusion. Bilateral plastic stenting was performed in eight patients, unilateral plastic stenting in two patients, bilateral SEMS in three patients, and balloon sweeping in one patient. The remaining 10 patients with stent occlusion underwent percutaneous transhepatic biliary drainage. Ultimately, endoscopic re-intervention failed in three patients because of entanglement of the stent assembly in the mesh cells of the in-
Table 3. Adverse Events Following Bilateral Stent-in-Stent Placement Using Large Cell Type Biliary Stents

\begin{tabular}{cc}
\hline \multicolumn{1}{c}{ Outcomes } & Data \\
\hline Early adverse events, $\mathrm{n}(\%)$ & $3 / 43(7.0)$ \\
Pancreatitis & 1 (Mild) \\
Cholangitis & 1 (Moderate) \\
Cholecystitis & 1 (Moderate) \\
Late adverse events, $\mathrm{n}(\%)$ & $24 / 38(63.2)^{*}$ \\
Stent occlusion & 24 \\
Multiple liver abscess & 1 \\
\hline
\end{tabular}

*One patient had both stent occlusion and liver abscess.

dwelling stents despite successful guidewire placement. In seven patients, percutaneous intervention was primarily performed owing to (1) newly developed additional intrahepatic duct dilation where SEMSs were not inserted, (2) a short life expectancy, or (3) a poor general condition of patients associated with sepsis following stent occlusion. Early adverse events occurred in three patients (7.0\%) (Table 3). Post-ERCP pancreatitis and cholangitis developed in one patient each. These patients were managed conservatively. Post-ERCP cholecystitis developed in one patient, and endoscopic ultrasound-guided gallbladder drainage was performed. There was no procedure-related mortality.

\section{DISCUSSION}

There are multiple barriers to successfully performing bilateral SIS placement for inoperable malignant hilar biliary obstruction, related to initial bilateral biliary cannulation and second guidewire cannulation of the desired contralateral intrahepatic duct via the indwelling first stent wire mesh and subsequent advancement of the second stent delivery system through the wire mesh and hilar biliary stricture. ${ }^{3,24}$ At the time of endoscopic reintervention, the guidewire should be advanced inside the indwelling stents and passed through the appropriate mesh cell of the indwelling stents, where it is expected that the subsequent stent assembly would not become entangled and could be advanced to the intrahepatic duct level. Therefore, the procedural steps for endoscopic re-intervention may be more challenging and complex than those of the initial SIS procedure.

To overcome these technical difficulties, an open-weave, cross-wired metal stent (Bonastent M-Hilar; Standard Sci Tech Inc., Seoul, Korea) $)^{5,10,15}$ and uniform large cell-type stent (Taewoong Corp. $)^{7-11}$ have been used and have shown excellent technical and clinical outcomes. In the studies regarding the efficacy of large cell-type stents for SIS placement, the reported technical success rate in a single session was $96 \%$ to $100 \%$, and the overall technical success was $100 \% .^{8-10}$ This high technical success rate has been explained by the unique mechanical properties of the large cell-type stent by previous studies. ${ }^{8-10}$ The 6-mm large cell can allow for easy insertion of the second stent 
through the first stent mesh and can facilitate further re-intervention following stent occlusion. The uniform cell structure of the large cell-type stent allows for flexibility in the placement of the second stent to adequately cover the distal portion of biliary stricture since the central portion of the stent should not necessarily be positioned in the hilar area.,11 Other mechanical advantages of large cell-type stents are their low axial force and enhanced radial force. ${ }^{7,11}$

However, we encountered several cases of technical failure in our study, unlike that in previous studies. ${ }^{8-10}$ In this study, we found that second stent failure can occur owing to the delivery sheath tip being caught in the hook-cross-type vertex of the mesh cell of the first stent. If the guidewire contacts this hookcross vertex of the mesh cell, the delivery sheath of the second stent over the guidewire could become entangled at that point because the vertex is relatively fixed and does not have an open weave. If this happens, the thicker nitinol wire interferes with the advancement of the stent assembly. In patients with a tight hilar stricture, another mesh cell negotiation to traverse a guidewire and stent assembly could be very challenging. In the current study, changing the mesh through which a guidewire was passed failed in all patients with second stent delivery entanglement in the first stent mesh vertex.

The median stent patency in this study (198 days) was comparable to that in previous studies regarding large cell-type stents $(105,157,202 \text { days })^{8-10}$ and open-weave, cross-wired stents (150 and 238 days) $)^{5,15}$; however, the stent occlusion rate in the current study (63.2\%) was somewhat higher than that in previous studies that considered large cell-type stents (42\%, $45.5 \%, 60 \%)^{8-10}$ and much higher than that in studies that examined open-weave, cross-wired stents (6\%, 30.8\%, 42.9\%). ${ }^{5,10,15}$ This result leads to a concern that the large cell-type stent may be vulnerable to stent occlusion by tumor ingrowth because of its wider mesh structure. ${ }^{7}$ However, a longer median patient survival (300 days) was noted in this study than in previously published studies regarding large cell-type stents (220 days) $)^{9,10}$ and open-weave, cross-wired stents (180 and 256 days). 5,10,15 Therefore, further large-scale studies are warranted to address this issue. In this study, the need for percutaneous transhepatic biliary drainage (41.6\%) was on the higher end of the range of the results published in previous studies regarding large celltype stents $(0 \%, 33.3 \%, 50 \%)^{8-10}$ or open-weave, cross-wired stents $(4.2 \%, 33.3 \%, 50 \%) .{ }^{5,10,15}$ However, most patients who required percutaneous transhepatic biliary drainage were not candidates for endoscopic re-intervention because of their poor medical condition and unstable vital signs or newly developed additional intrahepatic duct dilation where bilateral SEMSs were not inserted.

The strengths of the current study include its suggestion of the structural limitations of large cell-type stents as well as the relatively larger study population than that in previous studies on large cell-type stents. Nevertheless, the study also had sev- eral limitations: first, previous feasibility studies have already shown the technical and clinical results of the large cell-type stent. However, the negative impact of large cell-type stents for SIS placement described in the present study presents a good starting point for further studies to investigate whether large cell-type stents could be considered as a dedicated standard tool for SIS placement for palliation of inoperable malignant hilar strictures. Second, our study had a single-arm design without a comparison group; therefore, validation of our results is necessary through future prospective randomized trials that compare large cell-type stents with a new stent or a conventional open cell-type stent. Third, the four participating academic centers in the current study did not share a uniform clinical strategy for palliative anti-cancer treatment. This could have led to a bias in the stent patency and re-intervention results.

In conclusion, large cell-type stents for endoscopic bilateral SIS placement had acceptable rates of functional success and stent patency, if technically successful. However, the technical difficulty associated with entanglement of the second stent delivery sheath in the hook-cross-type vertex of the first stent may preclude large cell-type stents from being considered as a dedicated standard tool for SIS placement. A modification of the current design of large cell-type stents to address this issue may contribute to better technical success and a wider application of large cell-type stents.

\section{CONFLICTS OF INTEREST}

No potential conflict of interest relevant to this article was reported.

\section{REFERENCES}

1. Soehendra N, Reynders-Frederix V. Palliative bile duct drainage: a new endoscopic method of introducing a transpapillary drain. Endoscopy 1980;12:8-11.

2. Singhal D, van Gulik TM, Gouma DJ. Palliative management of hilar cholangiocarcinoma. Surg Oncol 2005;14:59-74.

3. Kim JH. Endoscopic stent placement in the palliation of malignant biliary obstruction. Clin Endosc 2011;44:76-86.

4. Lee JH, Kang DH, Kim JY, et al. Endoscopic bilateral metal stent placement for advanced hilar cholangiocarcinoma: a pilot study of a newly designed Y stent. Gastrointest Endosc 2007;66:364-369.

5. Park DH, Lee SS, Moon JH, et al. Newly designed stent for endoscopic bilateral stent-in-stent placement of metallic stents in patients with malignant hilar biliary strictures: multicenter prospective feasibility study (with videos). Gastrointest Endosc 2009;69: 1357-1360.

6. Hwang JC, Kim JH, Lim SG, Kim SS, Yoo BM, Cho SW. Y-shaped endoscopic bilateral metal stent placement for malignant hilar biliary obstruction: prospective long-term study. Scand J Gastroenterol 2011;46:326-332. 
7. Kogure H, Isayama H, Kawakubo K, et al. Endoscopic bilateral metallic stenting for malignant hilar obstruction using newly designed stents. J Hepatobiliary Pancreat Sci 2011;18:653-657.

8. Kogure H, Isayama H, Nakai Y, et al. Newly designed large cell Niti-S stent for malignant hilar biliary obstruction: a pilot study. Surg Endosc 2011;25:463-467.

9. Kogure H, Isayama H, Nakai Y, et al. High single-session success rate of endoscopic bilateral stent-in-stent placement with modified large cell Niti-S stents for malignant hilar biliary obstruction. Dig Endosc 2014;26:93-99.

10. Lee JM, Lee SH, Chung KH, et al. Small cell- versus large cell-sized metal stent in endoscopic bilateral stent-in-stent placement for malignant hilar biliary obstruction. Dig Endosc 2015;27:692-699.

11. Mukai T, Yasuda I, Isayama H, et al. Comparison of axial force and cell width of self-expandable metallic stents: which type of stent is better suited for hilar biliary strictures? J Hepatobiliary Pancreat Sci 2011;18:646-652.

12. Naitoh I, Hayashi K, Nakazawa T, et al. Side-by-side versus stentin-stent deployment in bilateral endoscopic metal stenting for malignant hilar biliary obstruction. Dig Dis Sci 2012;57:3279-3285.

13. Kim KM, Lee KH, Chung YH, et al. A comparison of bilateral stenting methods for malignant hilar biliary obstruction. Hepatogastroenterology 2012;59:341-346.

14. Law R, Baron TH. Bilateral metal stents for hilar biliary obstruction using a $6 \mathrm{Fr}$ delivery system: outcomes following bilateral and side-by-side stent deployment. Dig Dis Sci 2013;58:2667-2672.

15. Lee TH, Moon JH, Kim JH, et al. Primary and revision efficacy of cross-wired metallic stents for endoscopic bilateral stent-in-stent placement in malignant hilar biliary strictures. Endoscopy 2013; 45:106-113.

16. Kawakubo K, Kawakami H, Kuwatani M, et al. Single-step simultaneous side-by-side placement of a self-expandable metallic stent with a 6-Fr delivery system for unresectable malignant hilar biliary obstruction: a feasibility study. J Hepatobiliary Pancreat Sci 2015;22:151-155.

17. Dowsett JF, Vaira D, Hatfield AR, et al. Endoscopic biliary therapy using the combined percutaneous and endoscopic technique. Gastroenterology 1989;96:1180-1186.

18. Larghi A, Tringali A, Lecca PG, Giordano M, Costamagna G. Management of hilar biliary strictures. Am J Gastroenterol 2008;103: 458-473.

19. Vienne A, Hobeika E, Gouya H, et al. Prediction of drainage effectiveness during endoscopic stenting of malignant hilar strictures: the role of liver volume assessment. Gastrointest Endosc 2010;72: 728-735.

20. Chang WH, Kortan P, Haber GB. Outcome in patients with bifurcation tumors who undergo unilateral versus bilateral hepatic duct drainage. Gastrointest Endosc 1998;47:354-362.

21. Liberato MJ, Canena JM. Endoscopic stenting for hilar cholangiocarcinoma: efficacy of unilateral and bilateral placement of plastic and metal stents in a retrospective review of 480 patients. BMC Gastroenterol 2012;12:103.

22. Naitoh I, Ohara H, Nakazawa T, et al. Unilateral versus bilateral endoscopic metal stenting for malignant hilar biliary obstruction. J Gastroenterol Hepatol 2009;24:552-557.

23. Lee TH, Kim TH, Moon JH, et al. Bilateral versus unilateral placement of metal stents for inoperable high-grade malignant hilar biliary strictures: a multicenter, prospective, randomized study (with video). Gastrointest Endosc 2017;86:817-827.

24. Moon JH, Rerknimitr R, Kogure H, Nakai Y, Isayama H. Topic controversies in the endoscopic management of malignant hilar strictures using metal stent: side-by-side versus stent-in-stent techniques. J Hepatobiliary Pancreat Sci 2015;22:650-656.

25. Cotton PB, Lehman G, Vennes J, et al. Endoscopic sphincterotomy complications and their management: an attempt at consensus. Gastrointest Endosc 1991;37:383-393.

26. Kiriyama S, Takada T, Strasberg SM, et al. TG13 guidelines for diagnosis and severity grading of acute cholangitis (with videos). J Hepatobiliary Pancreat Sci 2013;20:24-34.

27. Yokoe M, Takada T, Strasberg SM, et al. TG13 diagnostic criteria and severity grading of acute cholecystitis (with videos). J Hepatobiliary Pancreat Sci 2013;20:35-46. 